\title{
New materials for modulators and switches in silicon photonics (Conference Presentation)
}

\author{
Dries Van Thourhout, Univ. Gent (Belgium); Marianna Pantouvaki, IMEC (Belgium); \\ Herbert D'Heer, Koen Alexander, Bart Kuyken, Univ. Gent (Belgium); Inge Asselberghs, \\ Steven Brems, Cedric Huyghebaert, IMEC (Belgium); Leili Abdollahi Shiramin, Univ. Gent \\ (Belgium); Chiara Alessandri, IMEC (Belgium); John P. George, Jeroen Beeckman, Univ. \\ Gent (Belgium); Min-Hsiang Hsu, Clement Merckling, Joris Van Campenhout, IMEC \\ (Belgium)
}

\begin{abstract}
In this presentation we will report on our recent work on new materials that can be monolithically integrated on high-index contrast silicon or silicon nitride photonic ICs to enhance their functionality. This includes graphene and other 2D-materials for realizing compact electro-absorption modulators and non-linear devices, ferroelectric materials for realizing phase modulators and adiabatic couplers for realizing bistable switches.
\end{abstract}

View presentation recording on the SPIE Digital Library:

http://dx.doi.org/10.1117/12.2253679.5393348497001 